\title{
Homogenous Grafted Poly(acrylic acid) Brushes on Ultra-flat Polydimethlysiloxane (PDMS) Films by UV Irradiation
}

Haijun Yang ${ }^{1,2}$, Zhengchi Hou,*

${ }^{1}$ Research Institute of Micro/Nano- Science and Techonology, Shanghai Jiao Tong University, Shanghai 200240, China

${ }^{2}$ Shanghai Institute Applied Physics, Chinese Academy of Sciences, Shanghai 201800, China

* Corresponding author:houzhengchi@sinap.ac.cn Phone: 86-21-3919 4936;Fax:86-21-59553476

\begin{abstract}
In this paper, hydrophilic poly(acrylic acid) (PAA) brushes were evenly grafted onto ultra-flat polydimethlysiloxane (PDMS) films by UV irradiation in a benzophenone (BP)/aceton/acrylic acid solution. The grafted films remain the elasticity of PDMS, which indicates the grafting reaction takes place at the superficial surface. But water affects the grafting depth much due to the good diffusion of monomers into the film.
\end{abstract}

Keywords: Graft, UV irradiation, Poly(acrylic acid), Polydimethlysiloxane (PDMS)

Citation: H. Yang, et al. Homogenous Grafted Poly(acrylic acid) Brushes on ultra-flat Polydimethlysiloxane (PDMS) Films by UV Irradiation. Nano Biomed. Eng. 2011, 3(1), 42-46. DOI: 10.5101/nbe.v3i1.p42-46.

\section{Introduction}

Polydimethylsiloxane (PDMS) has many unique properties, such as physiological inertness, good blood compatibility, low toxicity, transparency at $\mathrm{UV} / \mathrm{vis}$ wavelength, permeability to gases, low cost, reversible sealing to a lot of materials and so on. [1-3] Benefiting from these merits, it has been widely used in biomedicine, $[4,5]$ microfluidic chips, $[1,6,7]$ and soft lithography. [811] However, original PDMS is very hydrophobic with contact angle in the range of $90^{\circ}-102^{\circ}$, which leads to the accumulation of proteins [12,13] or other hydrophilic chemical regents $[6,12]$ on its surface, swelling by some organic solvents $[14,15]$, or repelling aqueous solution into its microchannels in fluidic chip. $[1,3,6,16]$

To make the surface of PDMS hydrophilic and maintain its elasticity, researchers have developed many methods, including physical adsorption, plasma treatment, plasma/ozone or UV/ozone oxidation, and surface grafting. [3, 14, 17-21] However, it has been proved that only the PDMS modified by surface grafting can maintain its hydrophilicity for a long time, [22] those modified by other methods were suffered hydrophobicity recovery due to the weak interaction with adsorbates or blended polymers, or the flexibility of PDMS chains which will embed the hydrophilic layer of oxide into the bulk. However, it's very difficult to graft polymer brushes on the surface of PDMS since the grafted polymer chains easily penetrates the network of PDMS chains due to 42 O'd its high flexibility. Although there are a lot of work on tailoring the PDMS surface by sequentially grafting poly(acrylic acid) (PAA) chains initiated by surface adsorbed photoinitiators to modify the hydrophobic channels in PDMS fluidic microchips [3, 20, 21, 23], they are focused on the improvement of the devices' performance. We and other peers have demonstrated that the trace of adsorbed photoinitiators affects the surface topography of the grafted PDMS films. [24, 25] And it's very hard to control the distribution of the grafted polymer chains.

In this work, acrylic acid was evenly grafted onto PDMS suface by UV irradiation. Different from the sequential UV graft method mentioned above, the photoinitiators was dissolved in the reaction solution in our experiments, which can get rid of the influence of the trace of adsorbed photoinitiators. In order to study the surface topography of the grafted PDMS, atomic force microscope (AFM) was adopted for the high-resolution profiling of surface morphology and nanostructure in tapping mode.

\section{Experimental section}

\subsection{Materials}

The Sylgard $184^{\mathrm{TM}}$ PDMS kit was purchased from Dow 
Corning and stored at $4^{\circ} \mathrm{C}$. Water $(18.2 \mathrm{M} \Omega \bullet \mathrm{cm})$ was treated with a Milli-Q integral water purification system (Millipore, USA). Acrylic acid (AAc) was purchased from Sinopharm Chemcial Reagent Co. Ltd (Shanghai, China) and purified by vacuum distillation before use. Benzophenone (BP), and acetone were also commercially obtained from Sinopharm Chemcial Reagent Co. Ltd (Shanghai, China) and used as received.

\subsection{Fabrication of ultra-flat PDMS films}

Flat PDMS films were fabricated as described previously. [26] Sylgard $184^{\mathrm{TM}}$ PDMS precursor was mixed thoroughly with its crosslinking agent at a ratio of 10:1 (w/w), and degassed by a vacuum pump. Then the mixture was cast on a freshly cleaved mica and degassed again to ensure good replication of the atomic flat surface of mica. Finally, the mixture was cured at $80^{\circ} \mathrm{C}$ for 4 hours. After peeling off from the mica surface, the PDMS films with ultra flat were obtained.

\subsection{Surface grafting poly(acrylic acid) (PAA) brushes by UV irradiation}

The UV graft of PAA onto PDMS surface was realized in one step. The reaction solution is composed of monomer (AAc), photoinitiator (BP), solvent (acetone) at the ratio of $0.5: 98: 1.5$. Before the reaction, the flat PDMS film was immersed in this solution and put in a temperature control system at $45^{\circ} \mathrm{C}$ for $30 \mathrm{~min}$. Then the reaction began by irradiating the solution under a lamp (1000W Xe). To ensure a uniform UV exposure, the Xe lamp was placed at the focus of a concave mirror. And the distance between the film and the center of the Xe lamp was about five centimeters. After reaction, the grafted films were washed in acetone three times to remove the remained BP and extracted in a Saxhlet apparatus by water for a week to remove the adsorbed monomers and polymers.

\subsection{Temperature control system}

The reaction temperature was controlled by immersion the reaction chamber in a water bath. Water with the temperature of $45^{\circ} \mathrm{C}$ was continuously flushed into the bath then drained to take the heat produced by the lamp away.

\subsection{Investigation of PDMS films by atomic force}
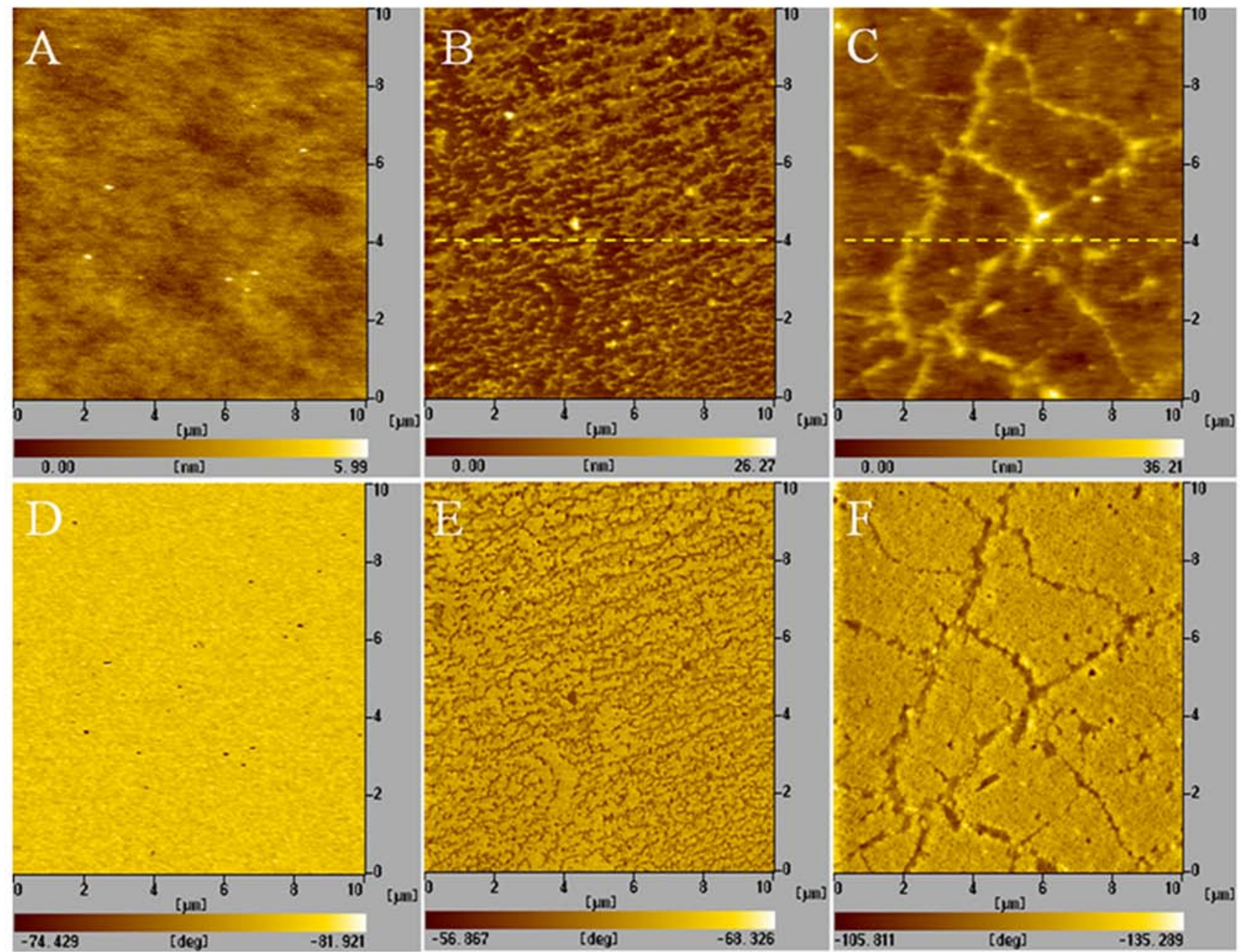

Fig. 1 AFM height and phase images of original ultra-flat PDMS films (A and D), grafted PDMS films with evenly distributed PAA brushes (B and $\mathrm{E})$, and grafted PDMS films affected by water in the reaction solution ( $\mathrm{C}$ and $\mathrm{F}$ ) 


\section{microscopy}

The topography of the dried PDMS films before and after grafting was measured by atomic force microscopy (SPA 400, Seiko Instruments, Japan) in tapping mode with the setpoint ratio of 0.85 . The rectangular silicon cantilever (NSC18/Cr-Au, MikroMasch, Russia) with a nominal constant of $3.5 \mathrm{~N} / \mathrm{m}$ and a typical resonant frequency of $75 \mathrm{kHz}$ was used for imaging.

\section{Results and Discussion}

All the height and phase images of PDMS film before and after grafting were shown in Fig. 1. The height images demonstrates their surface topography and the phase images which reflects variations in composition, adhesion, friction, viscoelasticity, and perhaps other properties. [27] From the scale bar in Fig. 1A, it can be found that the highest dots in this area are about 6 $\mathrm{nm}$ high. But the obvious contrast difference in phase image (Fig. 1D) manifests that these dots are something unknown rather than PDMS. Thus, if these dots are excluded, the height difference in the majority of this area is less than about $3 \mathrm{~nm}$. The ultra flatness of the original PDMS film may facilitate the discrimination of topography changes by AFM after UV grafting. Fig. 1B shows the surface topography of the PDMS film after grafted in an anhydrous solution composed of BP, AAc, and acetone. We can find that there are period wave-like nanostructures on the surface of this grafted film. These surface waves may be formed in the drying process, [28] since the topography of grafted layers scanned in air can be considered as the conformation of grafted chains in a poor solvent. [29] If there is water in the reaction solution, things go much different. Fig. 1C shows the surface topography of PDMS film grafted in the presence of water. It is quite different from the surface topography in Fig. 1B. We can find that the whole image was broken into many blocks by randomly linked ridges, just like a rimous dry paddy in Fig. 1F. [30] According to the information from Fig. 1C, we can also tell the different composition which respectively belongs to the ridges or the blocks. In scanning, the rigid places always appear higher than the soft ones under a constant force. [31] So, more PAA chains must have been grafted on the ridges than on the blocks, since PAA is rigid and PDMS is an elastomer. The great difference in the phase image (Fig. $1 F)$ can also prove this. For the places with more PAA chains (corresponding to the ridges in Fig. 1C), they should be more hydrophilic than other places. And the adhesion force between them and the AFM tip in scanning must be higher, then the great phase difference. Less the phase difference, more evenly distributed the grafted chains. In Fig. 1E, the phase difference is less than $12^{\circ}$, which may be resulted from the different thickness in the wave-like structure. And the grafted layer of PAA is evenly distributed in Fig. 1B.

We contribute the evenly distributed PAA chains on PDMS films to the steric hindrance effect of the grafted chains. In our acetone/BP/AAc reaction solution, acetone can slightly swell PDMS film. So, photoexcited benzophenone $\left(\mathrm{BP}^{*}\right)$ can diffuse into the surface and abstract hydrogen atoms from PDMS, producing the radicals required for grafting. [25] Since BP was dissolved in acetone in our experiments, the probability of initiating PAA grafting is identical all over the film surface; different from those initiated by the unenvenly adsorbed BP precipitation which determines the ultimate "footprint" of grafted polymer chains. [32] It's the precondition of obtaining uniform grafted PDMS films. After the initiation of grafting polymerization, the grafted chains become longer with the increase of the reaction time, like a living polymerization process. [33] However, the grafted PAA chains have limited compatibility with acetone, indicating that PAA with high molecular weight can't be dissolved in the reaction solution. The white precipitation on the film surface and in the solution can prove this. So, the grafted chains will precipitation on the film surface to form a grafted layer, which can block the diffusion of the monomer (AAc) into the bulk of PDMS
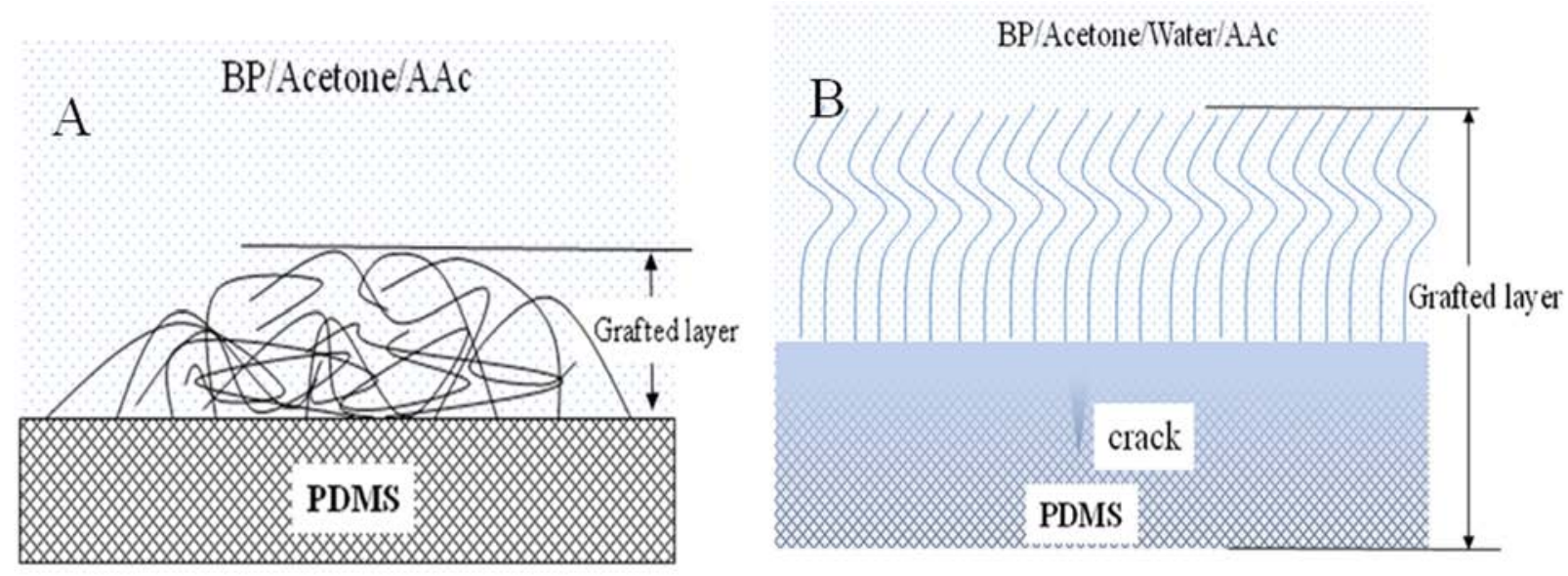

Fig. 2 Schematic illustration of UV grafting PAA onto ultra-flat PDMS films in different reaction solutions. (A) BP/Acetone/AAc solution, and (B) BP/Acetone/Water/AAc solution. 
film. In this case, grafting is most likely take place at the interface between polymer and solution, [34] as shown in Fig. 2A. And the elasticity of PDMS films is maintained.

When water exists in the reaction solution, the compatibility of grafted PAA chains with the hybrid acetone/water solvent is improved, reflected by the clearly transparent solution after reaction. So, the grafted PAA chains can stand on the PDMS surface and stretch into the solution, and the steric hindrance effect on grafting is eliminated. Monomers can easily penetrate the grafted layer and diffuse into the bulk of PDMS film. It's worth to point out that PDMS is transparent at UV-vis wavelength. Thus, the monomers which have diffused into the bulk of PDMS can also be grafted by the photoinitiator (BP). Grafting polymerization of PAA will take place in the bulk, as shown in Fig. 2B. But the monomers have many chances to be grafted on their diffusion trace. So, the grafting density is gradually decreased from the film surface to the center of the bulk, which will result in stress in the grafted PDMS film. If the stress exceeds the elasticity of the grafted film, cracks will come into being. And these cracks provide new interfaces for grafting. Monomers from the solution can easily reach and be grafted onto these places. Due to the close separation of the cracks, the density of grafted PAA chains is very high, and monomers diffused into the cracks most probably forms hydrogels with the grafted chains for the Tromasdorff effect. With the grafting reaction going on, the cracks becomes longer and longer, filled with PAA hydrogels. As a result, the content of rigid PAA chains in the cracks is higher than that on the blocks, which makes it looks like ridges in AFM height images. Since the PAA grafting takes place in the bulk of PDMS, the graft film becomes rigid. And the existence of the cracks in the bulk of PDMS makes it brittle and easily torn.

\section{Conclusions}

In summary, hydrophilic poly(acrylic acid) (PAA) brushes can be evenly grafted onto ultra-flat polydimethlysiloxane (PDMS) films by UV irradiation in an anhydrous benzophenone (BP)/aceton/acrylic acid solution. The grafting mainly takes place at the interface between the film surface and the reaction solution due to the steric hindrance of the grafted PAA layer with high molecular weight which can't be dissolved in the solution. If there is water in the reaction solution, the compatibility of the grafted PAA chains with the reaction solution is improved. In this case, monomers can diffuse into the PDMS film, and the grafting of PAA will take place in the bulk. Thus, the control on the monomer diffusion can provide us a good way to evenly graft PAA chains on hydrophobic PDMS films by UV grafting.

\section{Acknowledgements}

This work is supported by the grants from the National NSFC (No. 20776147, 10874198 and 90923041)

\section{References}

1. McDonald, J. C.; Duffy, D. C.; Anderson, J. R.; Chiu, D. T.; Wu, H.; Schueller, O. J. A.; Whitersides, G. M., Fabrication of microfluidic systems in poly(dimethylsiloxane). Electrophoresis 2000, 21, 27-40. doi:10.1002/(SICI)1522-2683(20000101)21:1<27::AID-ELPS2 7>3.0.CO;2-C

2. Gates, B. D.; Xu, Q.; Stewart, M.; Ryan, D.; Willson, C. G.; Whitesides, G. M., New Approaches to Nanofabrication: Molding, Printing, and Other Techniques. Chem. Rev. 2005, 105, 1171-1196. doi:10.1021/cr030076o

3. Hu, S.; Ren, X.; Bachman, M.; Sims, C. E.; Li, G. P.; Allbritton, N., Surface Modification of Poly(dimethylsiloxane) Microfluidic Devices by Ultraviolet Polymer Grafting. Anal. Chem. 2002, 74 (16), 4117-4123.

4. Abbasi, F.; Mirzadeh, H.; Katbab, A.-A., Modification of polysiloxane polymers for biomedical applications: a review. Polymer International 2001, 50, 1279-1287.doi:10.1002/pi.783

5. Chen, H.; Zhang, Z.; Chen, Y.; Brook, M. A.; Sheardown, H., Protein repellant silicone surfaces by covalent immobilization of poly (ethylene oxide). Biomaterials 2005, 26 (15), 2391-2399.doi:10.10 16/j.biomaterials.2004.07.068

6. Makamba, H.; Kim, J. H.; Lim, K.; Park, N.; Hahn, J. H., Surface modification of poly(dimethylsiloxane) microchannels. Electrophoresis 2003, 24, 3607-3619.doi:10.1002/elps.200305627

7. Ng, J. M. K.; Gitlin, I.; Stroock, A. D.; Whitesides, G. M., Components for integrated poly(dimethylsiloxane) microfluidic systems. Electrophoresis 2002, 23, 3461-3473.doi:10.1002/15222683(200210)23:20<3461::AID-ELPS3461>3.0.CO;2-8

8. Xia, Y.; Rogers, J. A.; Paul, K. E.; Whitesides, G. M., Unconventional Methods for Fabricating and Patterning Nanostructures. Chem. Rev. 1999, 99, 1823-1848.doi:10.1021/cr98 $0002 q$

9. Xia, Y.; Whitesides, G. M., Soft Lithography. Angew. Chem. Int. Ed. 1998, 37, 550-575.doi:10.1002/(SICI)1521-3773(19980316)37:5<5 50::AID-ANIE550>3.0.CO;2-G

10. Rogers, J. A.; Paul, K. E.; Jackman, R. J.; Whitesides, G. M., Using an elastomeric phase mask for sub-100 nm photolithography in the optical near field. Appl. Phys. Lett. 1997, 70 (20), 2658-2660. doi:1 $0.1063 / 1.118988$

11. Yang, H.; Wang, H.; Hou, Z.; Wang, P.; Li, B.; Li, J.; Hu, J., Fabrication and application of high quality poly(dimethylsiloxane) stamps by gamma ray irradiation. J. Mater. Chem. 2011, 21 (12), 4279-4285.doi:10.1039/c0jm03814h

12. Lahann, J. r.; Balcells, M.; Lu, H.; Rodon, T.; Jensen, K. F.; Langer, R., Reactive Polymer Coatings: A First Step toward Surface Engineering of Microfluidic Devices. Anal. Chem. 2003, 75, 2117 2122.doi:10.1021/ac020557s

13. Hu, S.; Ren, X.; Bachman, M.; Sims, C. E.; Li, G. P.; Allbritton, N., Cross-linked coatings for electrophoretic separations in poly (dimethylsiloxane) microchannels. Electrophoresis 2003, 24, 36793688.doi:10.1002/elps.200305592

14. Sadhu, V. B.; Perl, A. s.; Pe'ter, M. r.; Rozkiewicz, D. I.; Engbers, G.; Ravoo, B. J.; Reinhoudt, D. N.; Huskens, J., Surface Modification of Elastomeric Stamps for Microcontact Printing of Polar Inks. Langmuir 2007, 23, 6850-6855.doi:10.1021/la063657s

15. Trimbach, D. C.; Al-Hussein, M.; Jeu, W. H. d.; Decre', M.; Broer, D. J.; Bastiaansen, C. W. M., Hydrophilic Elastomers for Microcontact Printing of Polar Inks. Langmuir 2004, 20, 4738-4742. doi:10.1021/la049716o

16. Hartman, L. C.; Bessette, R. W.; Baier, R. E.; Meyer, A. E.; Wirth, J., Silicone rubber temporomandibular joint (TMJ) meniscal replacements: postimplant histopathologic and material evaluation. J. Biomed. Mater. Res. 1988, 22 (6), 475-484.doi:10.1002/jbm.820 220604

17. Lee, S.; Iten, R.; ller, M. M.; Spencer, N. D., Influence of Molecular Architecture on the Adsorption of Poly(ethylene oxide)-Poly (propylene oxide)-Poly(ethylene oxide) on PDMS Surfaces and Implications for Aqueous Lubrication. Macromolecules 2004, 37, 8349-8356.doi:10.1021/ma049076w

18. Choi, H.-G.; Zhang, Z.; Boccazzi, P.; Laibinis, P. E.; Sinskey, A. J.; Jensen, K. F., POLY(ETHYLENE GLYCOL) (PEG)- MODIFIED POLY(DIMETHYLSILOXANE) (PDMS) FOR PROTEIN- AND 
CELL-RESISTANT SURFACES IN MICROBIOREACTOR, 7th International Conference on Miniaturized Chemical and Blochemlcal Analysts Systems, October 5-9, 2003, Squaw Valley, Callfornla USA 2003, 1105-1108.

19. Berdichevsky, Y.; Khandurina, J.; Guttman, A.; Lo, Y.-H., UV/ozone modification of poly(dimethylsiloxane) microfluidic channels. Sensors and Actuators B 2004, 97, 402-408.doi:10.1016/j.snb.2 003.09 .022

20. Hu, S.; Ren, X.; Bachman, M.; Sims, C. E.; Li, G. P.; Allbritton, N. L. Surface-Directed, Graft Polymerization within Microfluidic Channels. Anal. Chem. 2004, 76, 1865-1870.doi:10.1021/ac049937z

21. Patrito, N.; McCague, C.; Chiang, S.; Norton, P. R.; Petersen, N. O., Photolithographically Patterned Surface Modification of Poly (dimethylsiloxane) via UV-Initiated Graft polymerization of Acrylates. Langmuir 2006, 22, 3453-3455.doi:10.1021/la0531751

22. Edmondson, S.; Osborne, V. L.; Huck, W. T. S., Polymer brushes via surface-initiated polymerizations. Chem. Soc. Rev. 2004, 33, 14. doi:10.1039/b210143m

23. Hu, S.; Ren, X.; Bachman, M.; Sims, C. E.; Li, G. P.; Allbritton, N. L., Tailoring the Surface Properties of Poly(dimethylsiloxane) Microfluidic Devices. Langmuir 2004, 20, 5569-5574.doi:10.1021/1 a0499741

24.Yang, H.; Hou, Z.; Hu, J., Surface modification of ultra-flat polydimethylsiloxane (PDMS) by UV grafted poly(acrylic acid) brushes. Journal of Applied Polymer Science 2011, in press.

25. Bhattacharya, A.; Misra, B. N., Grafting: a versatile means to modify polymers--Techniques, factors and applications. progress in polymer science 2004, 29, 767-814.

26. Yang, H.; Wang, P.; Wang, H.; Wang, X.; Zhang, F.; LI, B.; LI, H., Solvent-assisted nanotransfer printing on hydrophobic polymer substrate with high efficiency. Surface Review and Letters 2008,
27. http://www.chembio.uoguelph.ca/educmat/chm729/afm/moredet. htm.

28. Xi, H.-W.; Milner, S. T., Surface Waves on Polymer Brushes. Macromolecules 1996, 29, 4772-4776.doi:10.1021/ma951325g

29. Uchida, E.; Ikada, Y., Topography of Polymer Chains Grafted on a Polymer Surface Underwater. Macromolecules 1997, 30, 5464-5469. doi:10.1021/ma961660k

30. http://www.flickr.com/photos/88133845@N00/76721254/.

31.Banno, M.; Wu, Z.-Q.; Nagai, K.; Sakurai, S.-i.; Okoshi, K.; Yashima, E., Two-Dimensional Bilayer Smectic Ordering of Rigid Rod-Rod Helical Diblock Polyisocyanides. Macromolecules 2010, 43 (16), 6553-6561.doi:10.1021/ma1009116

32.Jones, D. M.; Brown, A. A.; Huck, W. T. S., Surface-Initiated Polymerizations in Aqueous Media: Effect of Initiator Density. Langmuir 2002, 18, 1265-1269.doi:10.1021/la011365f

33. Yamamoto, K.; Kyouzuka, S.-j.; Shimada, S., Generation and Molecular Motion of Grafted Polymethacrylate Chains on an Isotactic Polypropylene Film Surface. Macromolecules 2004, 37, 86-91.doi:10.1021/ma030334j

34. Ruckert, D.; Geuskens, G., surface modification of polymers-IV. grafting of acrylamide via an unexpected mechanism using a water soluble photo-initiator. Eur. Polym. J. 1996, 32 (2), 201-208. doi:10.1016/0014-3057(95)00136-0

Copyright:(c) $2011 \mathrm{H}$. Yang, et al. This is an openaccess article distributed under the terms of the Creative Commons Attribution License, which permits unrestricted use, distribution, and reproduction in any medium, provided the original author and source are credited. 